\title{
Corrected QT Interval Prolongation, Elevated Troponin, and Mortality in Hospitalized COVID-19 Patients
}

\author{
Rana Al-Zakharia, g(D), Muhammed KO Atere ${ }^{\mathrm{a}}$, William Limª, Mustafa Abdulrahman ${ }^{\mathrm{b}}$, Shahnaz Akhtarc, \\ Nicholas Sheets $^{\mathrm{d}, \mathrm{g}}$, Thomas Joyce ${ }^{\mathrm{d}}$, Veronika Stefanishina ${ }^{\mathrm{d}}$, Edmund Appiah-Kubie, \\ Philipa Owusu-Antwie, Jay Nfonoyim ${ }^{\mathrm{a}}$, Richard Grodman ${ }^{\mathrm{f}}$, Francesco Rotatorif
}

\begin{abstract}
Background: Severe acute respiratory syndrome coronavirus 2 (SARS-CoV-2) infection has risen to the level of a global pandemic. Growing evidence has proven the cardiac involvement in SARS$\mathrm{CoV}-2$ infection. This study aims to evaluate the ability of cardiovascular complications determined by elevated troponin and electrocardiogram findings (e.g., corrected QT interval (QTc)) in predicting the severity of SARS-CoV-2 infection among hospitalized patients.
\end{abstract}

Methods: This is a retrospective review of medical records of 800 patients, admitted to Richmond University Medical Center in Staten Island, NY, and tested positive for SARS-CoV-2 between March 1, 2020 and July 31,2020. A total of 339 patients met the study inclusion and exclusion criteria and were included in statistical analysis.

Results: Elevated serum troponin levels on admission statistically correlated with mortality in SARS-CoV-2 patients. Prolonged QTc was shown to have an independent statistically significant association with mortality among patients hospitalized with SARS-CoV-2.

Conclusions: Growing concern for cardiovascular sequelae of coronavirus disease 2019 (COVID-19) has prompted many researchers to investigate the role of cardiovascular complications in mortality due to SARS-CoV-2. Obtaining a simple electrocardiogram for hospital-

Manuscript submitted May 3, 2021, accepted May 17, 2021

Published online June 9, 2021

aDepartment of Medicine, Richmond University Medical Center, Staten Island, NY, USA

${ }^{b}$ Department of Medicine, Zucker School of Medicine at Mather/Northwell Health Mather Hospital, Port Jefferson, NY, USA

'Department of Clinical Research, Richmond University Medical Center, Staten Island, NY, USA

dSt. George's University School of Medicine, Grenada, West Indies

eSt. George's University, Building 300, 3500 Sunrise Highway, Great River, NY 11739, USA

fDepartment of Medicine-Cardiology, Richmond University Medical Center, Staten Island, NY, USA

gCorresponding Authors: Rana Al-Zakhari, Department of Medicine, Richmond University Medical Center, Staten Island, NY, USA. Email: rana alzakhari@yahoo.com; Nicholas Sheets, St. George's University School of Medicine, Grenada, West Indies. Email: nsheets@sgu.edu

doi: https://doi.org/10.14740/cr1276 ized patients with COVID-19 could provide an independent prognostic tool and prompt more coordinated treatment strategies to prevent mortality among patients hospitalized with COVID-19.

Keywords: SARS-CoV-2; QTc prolongation; Troponin; Coronavirus; Cardiovascular; QTc; Electrocardiogram; Mortality

\section{Introduction}

In Wuhan, Hubei province, China, the first reported cases of what would become known as coronavirus disease 2019 (COVID-19) were identified on December 8, 2019 [1]. On January 7, 2020, Chinese scientists first identified a novel coronavirus, severe acute respiratory syndrome coronavirus 2 (SARS-CoV-2), responsible for COVID-19 [2]. Transmission was identified as person-to-person, and spread was occurring at an unprecedented speed [3]. With rapid spread and severity of illness, the World Health Organization declared COVID-19 as a global pandemic [4]. At the time of writing in March 2021, 119 million cases of COVID-19 disease have been reported globally, with 2.7 million deaths reported. The case burden in the USA represents 29 million cases with 530 thousand moralities [5].

COVID-19 can present asymptomatically or with mild illness in $80 \%$ of cases, while the remaining $20 \%$ of cases can be classified as severe, requiring hospitalization with approximately 5\% needing admission to intensive care units [6]. Asymptomatic carriers have been implicated in the continued transmission of SARS-CoV-2. Severe presentation of the disease is thought to be due to significant lung parenchymal inflammation, showing a ground glass appearance on both computed tomography and chest X-ray [7]. In the presence of severe disease, COVID-19 pneumonia may evolve to acute respiratory distress syndrome (ARDS) and respiratory failure with progression to shock and multi-organ dysfunction syndrome through proposed destructive immune response with pro-inflammatory cytokines [6].

In this study, we will review the predictive ability of serum troponin and electrocardiogram (ECG) corrected QT interval (QTc) values in the setting of SARS-CoV-2 infection among hospitalized patients. Using this serum biomarker and ECG could provide prognostic value in the development of clinical 
management algorithms for patients hospitalized with COVID-19.

\section{Materials and Methods}

\section{Study population}

We conducted a retrospective review of medical records of patients admitted to Richmond University Medical Center (RUMC) in Staten Island, NY, USA, with laboratory-confirmed SARS-CoV-2 (positive results from nasopharyngeal sample via reverse transcriptase-polymerase chain reaction (RT-PCR)). Data were retrieved from 800 patients who were admitted between March 1, 2020 and July 31, 2020. Patients included in the study were male and female patients, ages greater than 18 years, had initial ECG performed on the day of admission, and tested positive for SARS-CoV-2 within the first $24 \mathrm{~h}$ of hospitalization. We excluded patients with chronic obstructive pulmonary disease, known arrhythmia (e.g., atrial fibrillation, atrial flutter), cardiac pacemaker (e.g., automatic implantable cardioverter defibrillator), congestive heart failure (CHF), heart murmurs, structural heart disease, hyperthyroidism, hypothyroidism, coronary artery disease, antiarrhythmic medications (e.g., procainamide, flecainide), echocardiogram findings of structural or valvular disease, or use of known medications before and during their hospitalization that implicated in QT prolongation (e.g., quinolones, macrolides, neuroleptics, antidepressants, antihistamines, methadone, and hydroxychloroquine) (Fig. 1).

This study was conducted in compliance with the all the applicable ethical standards of the responsible institution on human subjects, as well as in accordance with the Helsinki Declaration; and New York Medical College Institutional Review Board (IRB) approval was collected.

\section{Number of patients}

Patients from RUMC were screened and reviewed from electronic medical records and included based on exclusion criteria. Records were reviewed and individuals were included based on SARS-CoV-2 positive status. The enrollment criteria were maintained for the duration of the study. Based on inclusion and exclusion criteria, 339 patients were included in the study; and 401 patients were excluded.

\section{Data collection}

The data collected in this study were obtained from electronic medical records (patient demographics, patient expired status, patient ECG findings, QTc and patient's serum troponin and electrolytes levels). Data collected from patients were obtained in the first $24 \mathrm{~h}$ as hospitalized and defined as initial laboratory and investigative values. Normal values for QTc were defined as $<430 \mathrm{~ms}$ and $<450 \mathrm{~ms}$ for males and females, respectively. QTc measurements were automated collections from 12-lead ECG system. Troponin $<0.045 \mathrm{ng} / \mathrm{mL}$ was considered nor- mal. There was insufficient data to include B-type natriuretic peptide (BNP) in the statistical analysis of this study because initial BNP values (i.e., first $24 \mathrm{~h}$ on admission) were not obtained in the study population (Table 1).

\section{Statistical analysis}

Statistical analysis was performed using SPSS version 27.0 (IBM). The statistical differences were evaluated with Spearman rank correlation coefficient and Fisher exact test. Bivariate linear correlations analyses were assessed with the Spearman rank correlation coefficient. Probability value assessed whether the relationships between expired status and troponin, ECG abnormalities, and QTc are statistically significant. A consistent analysis of ECG and expired status was examined with Fisher exact test. For all analyses, a probability value of $\mathrm{P}<0.05$ was considered statistically significant. Values that retained statistical significance were reported with $95 \%$ confidence intervals (CIs). A Quade's ANCOVA was conducted to adjust for covariates; Stratification and multivariate analyses using Mantel-Haenszel (M-H) estimator and logistic regression were also implored to control for confounding variables such as calcium, magnesium, and potassium.

\section{Results}

A total of 339 patients were included in the study, 207 males and 132 females. Sex distribution skewed male in this sample population. Descriptive statistics revealed 126 of the subjects sampled were White, which comprised over a third of the subjects sampled $(37.2 \%) ; 27.7 \%$ of the subjects sampled were Black $(n=94)$, and $24.5 \%$ were Hispanic $(n=83)$, representing over half of the total sample $(52.2 \%)$. The lowest proportion of the total sample came from $5.3 \%$ of the subjects as Asian $(n=18)$ and $5.3 \%$ other $(n=18)$. Of note, this study was conducted in Staten Island, NY, with a majority White population (Table 2).

Of the 339 subjects, $99(27.2 \%)$ had expired status and 240 $(70.8 \%)$ did not have expired status. Through correlation analysis with expired status as the dependent variable, we found an association between several dependent variables. On the basis of direction, all independent variables were inversely related to expired status. This study broadly looked at two investigative modalities: ECG findings and serum troponin (Table 3).

\section{ECG findings}

The relationship between expired status and QTc showed a statistically significant correlation $(\mathrm{r}=-0.173, \mathrm{P}<0.05,95 \% \mathrm{CI}$ $(-0.277,-0.064))$. Additionally, we investigated if there was a relationship between expired status and abnormal ECG. Abnormal ECG findings were associated with expired status at a relatively lesser measure of association $(\mathrm{r}=-0.112, \mathrm{P}<0.05$, $95 \%$ CI $(-0.219,-0.003))$. In this study, a "normal ECG" was defined as normal sinus rhythm, and an "abnormal ECG" was 


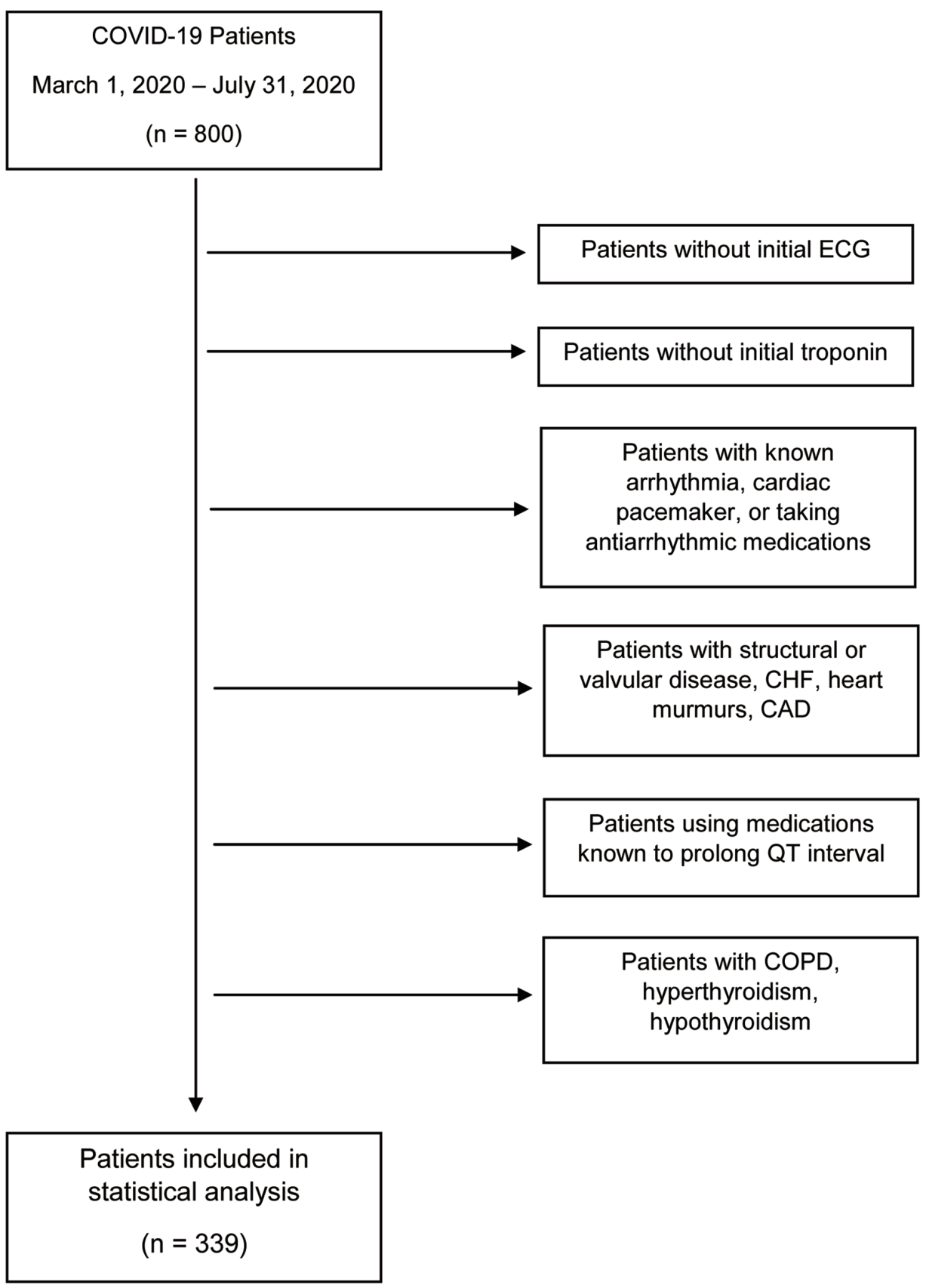

Figure 1. Flowchart of the enrollment of COVID-19 patients into study. COVID-19: coronavirus disease 2019; ECG: electrocardiogram; CHF: CHF: congestive heart failure; CAD: coronary artery disease; COPD: chronic obstructive pulmonary disease.

sinus tachycardia with or without premature atrial contractions or sinus tachycardia with or without premature ventricular contractions. Fisher exact test is 0.026 , which is consistent with the correlation analysis inference of an association between abnormal ECG parameters and expired status of subjects.

\section{Serum troponin}

A significant association was observed between expired status of the subjects and troponin levels $(\mathrm{r}=-0.228, \mathrm{P}<0.05,95 \%$ CI (-0.345, -0.104)). Increased values of troponin were correlated with expired status among subjects (Table 4).

\section{Discussion}

The impact of SARS-CoV-2 has been widely investigated, including targets for therapeutic intervention and diagnosis. The abundance of reports and data of patients admitted for pulmo- 
Table 1. QT and Troponin Measurements

\begin{tabular}{clll}
\hline & $\begin{array}{l}\text { Both males } \\
\text { and females }\end{array}$ & Males & Females \\
\hline QT interval & & & \\
\hline Normal & $\leq 430 \mathrm{~ms}$ & $\leq 430 \mathrm{~ms}$ & $\leq 450 \mathrm{~ms}$ \\
Prolonged & $>470 \mathrm{~ms}$ & $>430 \mathrm{~ms}$ & $>450 \mathrm{~ms}$ \\
Troponin & & & \\
\hline Normal & $\leq 0.5 \mathrm{ng} / \mathrm{mL}$ & - & - \\
\hline Elevated & $>0.5 \mathrm{ng} / \mathrm{mL}$ & - & - \\
\hline
\end{tabular}

Table 2. Distribution of Participants

\begin{tabular}{lll}
\hline & Frequency & Percent \\
\hline Expired & 99 & 29.2 \\
Unexpired & 240 & 70.8 \\
Male & 207 & 61.1 \\
Female & 132 & 38.9 \\
White & 126 & 37.2 \\
Hispanic & 83 & 24.5 \\
Black & 94 & 27.7 \\
Asian & 18 & 5.3 \\
Other & 18 & 5.3 \\
\hline
\end{tabular}

nary symptoms and dying of cardiovascular complications highlight the need for early detection, evaluation, and prognostication in these patients. A large, multihospital retrospective cohort of nearly 3,000 patients revealed myocardial injury occurred in $36 \%$ of patients [8]. Arrhythmias due to systemic illness secondary to COVID-19 can present unexpectedly [9]. Early identification of increased risk of adverse cardiovascular disease processes may have a profound impact on the identification and management of these patients hospitalized with COVID-19. In our cohort we found a statistically significant correlation between expired status of subjects (mortality) and several independent variables, including QTc, abnormal ECG
Table 4. Comparison of Elevated Troponin, Prolonged QTC, and Expired Status Among Black and White Patients

\begin{tabular}{llllll}
\cline { 2 - 3 } & \multicolumn{2}{c}{ Black } & & \multicolumn{2}{c}{ White } \\
\cline { 2 - 3 } \cline { 5 - 6 } Normal troponin & $50.0 \%$ & 30 & & $58.3 \%$ & 49 \\
Elevated troponin & $50.0 \%$ & 30 & & $42.7 \%$ & 35 \\
Normal QTc & $42.6 \%$ & 40 & & $45.2 \%$ & 57 \\
Prolonged QTc & $57.4 \%$ & 54 & & $54.8 \%$ & 69 \\
Expired & $34.0 \%$ & 32 & $30.2 \%$ & 38 \\
Not expired & $66.0 \%$ & 62 & $69.8 \%$ & 88 \\
\hline
\end{tabular}

Sample size for QT interval and expired status was 220 (94 Black patients and 126 White patients). The sample size for troponin was 144 (60 Black patients and 84 White patients). The sample size for troponin was smaller due to missing data. Initial troponin levels were not obtained in all. QTc: corrected QT interval.

findings, and serum troponin. Our findings are consistent with current literatures that demonstrate cardiovascular involvement in COVID-19 pathophysiology and mortality while correcting for pre-existing cardiovascular pathology.

It has been accepted that COVID-19 disease can include cardiovascular disease processes. There is an increased risk of mortality from COVID-19 infection in association with risk factors such as diabetes, hypertension, obesity, elderly individuals, and male sex [10]. The most common cardiac COVID-19 disease manifestations include acute myocardial injury. These acute injuries can result from direct myocardial injury, systemic inflammation, and myocardial oxygen demand supply mismatch. Other possible complications include acute coronary event, left ventricular systolic dysfunction, heart failure, arrhythmias, and other complications [11]. There are currently three suspected mechanisms of cardiovascular pathophysiology. The first describes a cytokine-induced systemic inflammatory response resulting in cardiac dysfunction. The second describes SARS-CoV-2 affecting small blood vessels resulting in injury to myocardial tissue leading to possible arrhythmia. The third proposed mechanism is that SARS-CoV-2 induces myocardial ischemia, leading to immunity-induced cardiac dysfunction with possible ventricular arrhythmia development

Table 3. Results of Statistical Analyses

\begin{tabular}{lll} 
& Spearman's rho & P value (95\% CI) \\
\hline Expired status and sex & 0.087 & $0.109(-0.023,0.195)$ \\
Expired status and age & -0.273 & $0.000(-0.372,-0.168)$ \\
Expired status and race & 0.088 & $0.105(-0.022,0.196)$ \\
Expired status and ECG & -0.112 & $<0.05(-0.219,-0.003)$ \\
Expired status and QTc & -0.173 & $0.001(-0.277,-0.064)$ \\
Expired status and calcium & 0.134 & $0.014(0.025,0.240)$ \\
Expired status and potassium & -0.156 & $0.004(-0.261,-0.047)$ \\
Expired status and troponin & -0.228 & $0.000(-0.345,-0.104)$ \\
\hline
\end{tabular}

The table showed the correlation coefficient of the variables and their independent correlation to expired status in 339 subjects. All analyses are reported at P < 0.05 statistical significance with $95 \% \mathrm{Cl}$. Cl: confidence interval. ECG: electrocardiogram; QTc: corrected QT interval. 
[6]. COVID-19 induced arrhythmias are thought to arise from metabolic disturbances, hypoxia, direct viral myocardial injury, and heightened inflammation [11]. There is growing evidence that the inflammatory "cytokine storm" seen in COVID-19 is due to an increased production of interleukin-6 (IL-6), which has contributed to possible prolongation of the QT interval and sequentially torsades de pointes (TdP) [12].

The depolarization of the ventricle, denoted as the QRS complex on an ECG to the T wave (electrically representing ventricular repolarization) is known as the QT interval. A normal QT interval is defined as $350-450 \mathrm{~ms}$ in males and 360 - $460 \mathrm{~ms}$ in females [13]. If there is a delay in the repolarization of the ventricles it may represent an electrical disturbance on ECG and can be referred to as a prolonged QT interval. Prolonged QT interval has been associated with an increased risk of coronary heart disease, arrhythmias, sudden cardiac death and other cardiological pathologies [14]. Delayed ventricular repolarization has been demonstrated to enable early depolarizations, thus resulting in the fatal arrhythmia TdP [15]. Arrhythmias may occur due to systemic illnesses, drug interactions, as well as direct viral effects of certain pathogens. In patients with SARS-CoV-2, cardiac arrhythmias are increasingly identified. The pathogenesis of the resulting arrhythmias among these patients may be due to viral involvement in the lung tissue resulting in hypoxia, myocarditis, cardiac ischemia, electrolyte disturbances, abnormal host immune response, and side effects of various pharmacological therapies used in treatment of the viral infection [16].

A key finding was the association between QTc prolongation and mortality. Previous studies have evaluated the relationship between QTc prolongation and medication treatment in the setting of COVID-19, but they have mostly attributed the increased QT interval to medication treatment with chloroquine/ hydroxychloroquine in patients with no prior QTc abnormalities in baseline ECG [17]. In this study, pharmacological agents that have been known to prolong the QT interval were excluded from the sample population. Thus, this study investigates the possible role of SARS-CoV-2 infection on QTc prolongation while controlling for confounding variables. A statistically significant monotonic relationship was identified between expired status of subjects and QTc. Additionally, our study found, to a lesser degree, a correlation between abnormal ECG (e.g., sinus tachycardia with or without premature atrial contractions, sinus tachycardia with or without premature ventricular contractions) and expired status of subjects. With ECG being used as a tool throughout the world, this could have beneficial implications for the diagnostic and prognostic value of obtaining an ECG in patients hospitalized with COVID-19. Early identification of abnormal ECG findings in the setting of COVID-19 could allow for early escalation in treatment, thus reducing cardiovascular mortality among COVID-19 hospitalized patients. Preexisting cardiovascular comorbidities in the setting of severe COVID-19 infection have been shown to have a case fatality rate of $10.5 \%$ [18]. Because of the extensive exclusion criteria in this study, we can postulate that the ECG findings observed are most likely due to infection with SARS-CoV-2, whether it be via systemic inflammation or direct myocardial injury.

Our data revealed the greatest correlation between elevated troponin levels and expired status of subjects. A recent systematic review demonstrated that cardiac specific biomarkers, such as troponin and N-terminal-pro-BNP (NT-pro-BNP), were associated with increased mortality and underscored their value as prognostic indicators [18]. Lala et al found that even low-level concentrations of troponin were associated with three-fold increased risk of mortality [8]. Our findings demonstrate an association with increased troponin levels consistent with the current literature. A recent meta-analysis demonstrated that those with severe COVID-19 infection and nonsurvivors had a much greater burden of CHF, and that these patients were at an increased risk of acute cardiac injury and rhythm irregularities, which was measured via increased levels of cardiac biomarkers [19]. However, our study sought to exclude those with pre-existing CHF; therefore infection with SARS-CoV-2 could have a greater role on myocardial injury than previously thought.

\section{Limitations}

There are a few limitations to this study. First, the study population may be prone to sampling bias. A total of 800 patients were found to be eligible, but 401 patients had to be excluded due to the absence of the initial ECG and serum biomarkers. Because of these exclusions, the sample used may not be an accurate representation of the target population. Therefore, extrapolation of the data to all hospitalized patients with COVID-19 should be guarded. Second, the demographics of the subjects included in statistical analysis showed that over half of the sample was Black or Hispanic. The population in Staten Island, NY is predominantly White; therefore, the data may not be an accurate representation of the target population. However, Black individuals have been disproportionately affected by COVID-19 infection and rate of death [20]. This observed trend could account for a higher number of Black individuals included in the overall sample, despite Staten Island being a predominantly White borough of New York City. Initial troponin values were not collected in as many subjects as initial ECG; making the sample size used for statistical analysis smaller for initial troponin values. Finally, QTc was measured by an automated ECG system in this study, and this measurement could be prone to computer error.

\section{Future considerations}

Future studies could further investigate the benefit of clinical management algorithms of abnormal ECG findings and serum troponin in the setting of SARS-CoV-2 infection. If future studies can demonstrate advantage of these as a prognostic model, then the cost of obtaining these tests may be justified. The growing evidence of cardiovascular-related mortality among COVID-19 patients suggests close monitoring of cardiac biomarkers and electrophysiology. With vaccination efforts underway, it will be prudent to follow long-term cardiovascular sequelae in patients with a history of the novel SARS-CoV-2 infection.

Growing concern for cardiovascular sequelae of COVID-19 has encouraged many researchers to investigate the role 
of SARS-CoV-2 in cardiovascular complications and mortality. Prolonged QTc and abnormal ECG findings were associated with increased mortality among patients hospitalized with COVID-19. Obtaining a simple ECG for hospitalized patients with COVID-19 could provide an independent prognostic tool and prompt more coordinated treatment strategy to prevent mortality among patients hospitalized with COVID-19. Elevated serum levels of troponin were also associated with higher mortality among patients hospitalized with COVID-19.

\section{Acknowledgments}

The authors wish to thank Zohaib Khan , BS , Adrew Malek, BS, Reema Iqbal, BS, Sukhdev Singh, BS, Jack Demirjian, BS, Pareena Singh, BS, Eugenie Le Roux, BS, Ecaterina Komarnitsky, BS, Jeffery Cesare, BS, Allison Foster, BS, Aisha Siddiqui, BS, Krisha Arora, BS, Zeynab Rana, BS for assisting in gathering patients data.

\section{Financial Disclosure}

None to declare.

\section{Conflict of Interest}

None to declare.

\section{Informed Consent}

Not applicable.

\section{Author Contributions}

RA and MKOA are primary authors; designed the study, engaged in data collection, reviewed the literature, and drafted the manuscript. POA and EA analyzed and interpreted the data. WL, MA, SA, TJ, VS and NS engaged in data collection, data interpretation, and contributed to drafts of the manuscript. JN is the primary investigator. FR and RG provided critical reviews of the manuscript.

\section{Data Availability}

The authors declare that data supporting the findings of this study are available within the article.

\section{Abbreviations}

ARDS: acute respiratory distress syndrome; ECG: electrocardiogram; RT-PCR: reverse transcriptase-polymerase chain reaction; BNP: B-type natriuretic peptide; $\mathrm{CI}$ : confidence interval; IL-6: interleukin-6; TdP: torsades de pointes; $\mathrm{CHF}$ : congestive heart failure

\section{References}

1. Guzik TJ, Mohiddin SA, Dimarco A, Patel V, Savvatis K, Marelli-Berg FM, Madhur MS, et al. COVID-19 and the cardiovascular system: implications for risk assessment, diagnosis, and treatment options. Cardiovasc Res. 2020;116(10):1666-1687.

2. Wang C, Horby PW, Hayden FG, Gao GF. A novel coronavirus outbreak of global health concern. Lancet. 2020;395(10223):470-473.

3. Domling A, Gao L. Chemistry and biology of SARSCoV-2. Chem. 2020;6(6):1283-1295.

4. Peirlinck M, Linka K, Sahli Costabal F, Kuhl E. Outbreak dynamics of COVID-19 in China and the United States. Biomech Model Mechanobiol. 2020;19(6):2179-2193.

5. Dong E, Du H, Gardner L. An interactive web-based dashboard to track COVID-19 in real time. Lancet Infect Dis. 2020;20(5):533-534.

6. Tsatsakis A, Calina D, Falzone L, Petrakis D, Mitrut R, Siokas V, Pennisi M, et al. SARS-CoV-2 pathophysiology and its clinical implications: An integrative overview of the pharmacotherapeutic management of COVID-19. Food Chem Toxicol. 2020;146:111769.

7. Dhama K, Khan S, Tiwari R, Sircar S, Bhat S, Malik YS, Singh KP, et al. Coronavirus disease 2019-COVID-19. Clin Microbiol Rev. 2020;33(4).

8. Lala A, Johnson KW, Januzzi JL, Russak AJ, Paranjpe I, Richter F, Zhao S, et al. Prevalence and impact of myocardial injury in patients hospitalized with COVID-19 infection. J Am Coll Cardiol. 2020;76(5):533-546.

9. Bugert CL, Kwiat V, Valera IC, Bugert JJ, Parvatiyar MS. Cardiovascular injury due to SARS-CoV-2. Curr Clin Microbiol Rep. 2021:1-11.

10. Richardson S, Hirsch JS, Narasimhan M, Crawford JM, McGinn T, Davidson KW, the Northwell C-RC, et al. Presenting characteristics, comorbidities, and outcomes among 5700 patients hospitalized with COVID-19 in the New York City Area. JAMA. 2020;323(20):2052-2059.

11. Gerstein NS, Venkataramani R, Goumas AM, Chapman NN, Deriy L. COVID-19-related cardiovascular disease and practical considerations for perioperative clinicians. Semin Cardiothorac Vasc Anesth. 2020;24(4):293-303.

12. Lazzerini PE, Laghi-Pasini F, Acampa M, Boutjdir M, Leopoldo Capecchi P. IL-6 (Interleukin 6) blockade and heart rate corrected QT interval prolongation in COVID-19. Circ Arrhythm Electrophysiol. 2020;13(9):e008791.

13. Postema PG, Wilde AA. The measurement of the QT interval. Curr Cardiol Rev. 2014;10(3):287-294.

14. Leotta G, Maule S, Rabbia F, Del Colle S, Tredici M, Canade A, Verhovez A, et al. Relationship between QT interval and cardiovascular risk factors in healthy young subjects. J Hum Hypertens. 2005;19(8):623-627.

15. Jankelson L, Karam G, Becker ML, Chinitz LA, Tsai MC. QT prolongation, torsades de pointes, and sudden 
death with short courses of chloroquine or hydroxychloroquine as used in COVID-19: A systematic review. Heart Rhythm. 2020;17(9):1472-1479.

16. Dherange P, Lang J, Qian P, Oberfeld B, Sauer WH, Koplan B, Tedrow U. Arrhythmias and COVID-19: A Review. JACC Clin Electrophysiol. 2020;6(9):1193-1204.

17. Mehraeen E, Seyed Alinaghi SA, Nowroozi A, Dadras O, Alilou S, Shobeiri P, Behnezhad F, et al. A systematic review of ECG findings in patients with COVID-19. Indian Heart J. 2020;72(6):500-507.
18. Shafi AMA, Shaikh SA, Shirke MM, Iddawela S, Harky A. Cardiac manifestations in COVID-19 patients-A systematic review. J Card Surg. 2020;35(8):1988-2008.

19. Sinclair JE, Zhu Y, Xu G, Ma W, Shi H, Ma KL, Cao CF, et al. A meta-analysis on the role of pre-existing chronic disease in the cardiac complications of SARS-CoV-2 infection. iScience. 2021;24(4):102264.

20. Golestaneh L, Neugarten J, Fisher M, Billett HH, Gil MR, Johns T, Yunes M, et al. The association of race and COVID-19 mortality. EClinicalMedicine. 2020;25:100455. 